\title{
Cyclic changes of the ovarian surface epithelium in the rat
}

\author{
M Gaytán, M A Sánchez, C Morales ${ }^{1}$, C Bellido, Y Millán², J Martín de las Mulas², \\ J E Sánchez-Criado and F Gaytán
}

Department of Cell Biology, Physiology and Immunology, ${ }^{7}$ Department of Pathology and ${ }^{2}$ Department of Comparative Pathology, University of Córdoba, Spain

Correspondence should be addressed to F Gaytán, Department of Cell Biology, Physiology and Immunology School of Medicine Avda Menendez-Pidals/n, 14004-Cordoba, Spain; Email: bc1galuf@uco.es

\begin{abstract}
The ovarian surface epithelium (OSE) plays pivotal roles during ovulation and postovulatory wound repair. In this paper we describe the proliferative activity of the OSE through the estrous cycle in adult cycling rats, by immunohistochemical detection of DNA-incorporated bromodeoxyuridine (BrdU). Immunohistochemical detection of estrogen receptor $\alpha$ (ER $\alpha)$ and progesterone receptor was also performed. The cycle of the OSE consists of a proliferative phase (that lasts for two consecutive estrous cycles) and a quiescent phase of variable duration. Cyclic changes in the OSE were related to the underlying ovarian structure. OSE areas covering growing follicles entered into the proliferative phase during the transition from proestrus to estrus, with the appearance of fast-growing class 1 follicles, destined to ovulate at the end of the current estrous cycle. A labeling index (after pulse-labeling BrdU treatment) of about $7 \%$ was maintained throughout the estrous cycle in parallel to follicle growth. Cumulative BrdU-labeling (after daily BrdU treatment) indicated that about 1/3 of the total OSE cell proliferation was related to follicle growth. Following ovulation, OSE cells covering newly-formed corpora lutea showed a labeling index of about $\mathbf{5 0} \%$ that decreased through metestrus and diestrus (about $13 \%$ and $3 \%$, respectively), returning to basal levels by proestrus. Cumulative BrdU-labeling indicated that about $2 / 3$ of the total proliferative activity was related to ovulation repair/luteinization. The remaining OSE covering ovarian stroma or structurally regressing corpora lutea of previous cycles showed negligible BrdU labeling. The equivalent proliferative activity found in the OSE covering newly-formed corpora lutea in indomethacin-treated rats lacking rupture of the OSE at the apex, demonstrated that ovulation-triggered proliferation was not dependent on the loss of integrity of the OSE at the ovulation site. OSE cells expressed ER $\alpha$ throughout the cycle, but no differential expression was found between proliferating and quiescent OSE areas. On the contrary, OSE cells did not express PR at any time of the cycle. These data indicate the existence of a cycle of the OSE, related to the cyclic changes in the underlying ovarian structure and strongly suggest that the proliferative activity of the OSE is regulated by local microenvironmental rather than by systemic factors.

Reproduction (2005) 129 311-321
\end{abstract}

\section{Introduction}

The ovary is surrounded by a layer of cells supported by a basal lamina, that constitutes the OSE. The OSE is embryologically derived from the celomic epithelium and varies morphologically from simple squamous to cuboidal to pseudostratified columnar (Auersperg et al. 2001). Due to its inconspicuous appearance in histological sections and to the apparent lack of significant functions, the OSE has received less attention than other ovarian tissue compartments. Aside from the growing interest when it became apparent that almost $90 \%$ of ovarian cancers (the most lethal among gynecological malignancies) arise from the OSE (Godwin et al. 1993, Auersperg et al. 2001), initial interest in the OSE was due to its participation in ovulation. As follicle rupture occurs at the apex - that is at the follicle side facing the ovarian surface - local disruption and subsequent repair of the OSE has to occur during ovulation. However, the role of the OSE in ovulation is still controversial. Although early studies proposed an active role for the OSE in the ovulatory process (Bjersing and Cajander 1975), this notion was discounted thereafter by the observation that ovulation still occurred in some rabbit follicles after OSE scrapping (Rawson and Espey 1977). However, more recent studies strongly suggest that the OSE plays an active role in ovulation, participating in the proteolytic breakdown of the basement membrane and the ovarian tunica albuginea (Murdoch and McDonnel 2002), thus contributing to the spatial targeting of follicle rupture at the apex (Gaytán et al. 2003). 
As OSE rupture is an obligate component of ovulation, proliferation of OSE cells plays a pivotal role during ovulatory wound repair. Reparative mitogenic activity of OSE cells is currently considered as a main factor favouring accumulation of mutagenic events leading to OSE cell transformation and ovarian cancer (Murdoch et al. 2001, Murdoch and McDonnel 2002). In this context, knowledge of the mechanisms regulating OSE cell proliferation is essential to the understanding of OSE biology either in physiological or pathophysiological conditions. In spite of its morphological simplicity, recent studies have pointed out the complexity of OSE cell biology and functional regulation (Auersperg et al. 2001). As the ovary is under the control of gonadotropins and steroids, these hormones are strong candidates to regulate the OSE. The potential for gonadotropins and steroids to regulate OSE cell proliferation is suggested by the demonstration of receptors for these hormones in the OSE of several species (HildPetito et al. 1988, Zheng et al. 1996, Pelletier et al. 2000, Kuroda et al. 2001, Okada et al. 2002), although interspecies variations have been reported (Hess et al. 1999, Pelletier et al. 2000). In general, in vivo studies have reported that gonadotropins (Davies et al. 1999, Hess et al. 1999, Stewart et al. 2004) and estrogens (Adams and Auersperg 1983, Bai et al. 2000) stimulate OSE cell proliferation. However, in vitro studies have provided variable results. Whereas some studies have reported that gonadotropins and estrogens stimulate the proliferative activity of OSE cells in culture (Bai et al. 2000, Murdoch and van Kirk 2002), other studies having found a lack of mitogenic effects of gonadotropins and steroids in isolated OSE cells (Karlan et al. 1995, Syed et al. 2001, Wright et al. 2002), suggested that the effects of gonadotropin and estrogens could be mediated by the local release of growth-promoting factors.

Most in vivo studies have explored the proliferative activity of the OSE at the follicle apex, in relation to ovulation wound repair (Osterholzer et al. 1985, Beller et al. 1995), or after gonadotropin stimulation (Beller et al. 1995, Davies et al. 1999, Stewart et al. 2004). However, the proliferative activity of the OSE throughout the estrous cycle has received little attention, and a comprehensive view of the cyclic changes in the OSE is lacking. In order to examine further the regulation of the OSE cell proliferation, we analysed the proliferative activity of the OSE in adult cycling rats, in relation to the main ovarian reproductive events, such as follicle growth, ovulation, luteogenesis and luteolysis. We describe herein cyclic changes in the proliferative activity of OSE cells, related to the cyclic changes in the underlying ovarian structure, by using immunohistochemical detection of DNA-incorporated bromodeoxyuridine, as a marker of OSE cell proliferation, as well as the expression of ER $\alpha$ and progesterone receptor (PR) in OSE cells throughout the estrous cycle.

\section{Materials and Methods}

\section{Animals and drugs}

Adult cycling Wistar rats were used. The animals were maintained under controlled light $(14 \mathrm{~h}$ light/10 h darkness) and temperature $\left(22^{\circ} \mathrm{C}\right)$ conditions and had free access to pelleted food and tap water. The estrous cycle was monitored by taking daily vaginal smears. Only rats displaying at least two consecutive four-day estrous cycles were used. The experiments were carried out in agreement with the National Institute of Health's Guide for the Care and Use of Laboratory Animals and were approved by the ethic committee of the University of Cordoba. Bromodeoxyuridine and indomethacin (Sigma Chemical Co., St Louis, MO, USA) as well as monoclonal antibodies against BrdU (Dako Diagnostica, Hamburg, Germany), $\mathrm{ER} \alpha$ (Dakocytomation, Glostrup, Denmark) and PR (Immunotech, Marseille, France) were used.

\section{Experimental designs}

\section{BrdU pulse-labeling during the estrous cycle and pregnancy}

Cycling rats in the different stages of the estrous cycle (five animals per group) were used. One hour before death, the animals were injected ip with $0.5 \mathrm{mg} / \mathrm{Kg}$ bw of BrdU in $0.1 \mathrm{M}$ Tris- $\mathrm{HCl}$ buffer $(\mathrm{pH} 7.2)$. Animals were killed by decapitation at 02:00, 07:00 and 12:00 h in estrus, at 09:00 $\mathrm{h}$ in metestrus and diestrus, and at 09:00, 18:30 and 21:00 $\mathrm{h}$ in proestrus. The ovaries, including ovarian bursa and periovarian fat pad were removed and fixed for at least $24 \mathrm{~h}$ in Bouin-Hollande's fluid before being processed for paraffin embedding. Some additional rats (3 per time point) were injected with $\mathrm{BrdU}$ as in the previous experiment on days $6,12,15$, and 21 of pregnancy (the first day in which sperm was found in vaginal smears was considered as day 1 of pregnancy), and the ovaries were processed as described above.

\section{Indomethacin-treated cycling rats}

Adult cycling rats were injected with $1 \mathrm{mg} / \mathrm{Kg}$ bw of indomethacin or vehicle (olive oil) at 12:00 $\mathrm{h}$ on the morning of proestrus, and killed at 09:00 h on the day of estrus. This dosage and time schedule have been previously reported to induce aberrant ovulations, and several types of newly-formed corpora lutea showing ruptured or unruptured OSE can be found (Gaytán et al. 2003). One hour before death, the animals received an ip injection of $\mathrm{BrdU}$ as in previous experiments. The ovaries of five animals per group were processed as described above.

\section{Cumulative BrdU-labeling}

In order to analyse the cumulative proliferative activity related to follicle growth and ovulation repair/luteinization, cycling rats (5 animals) were injected daily with $0.5 \mathrm{mg} / \mathrm{Kg}$ 
bw of BrdU at 11:00 h from estrus to proestrus. To avoid repetitive ip injections, BrdU was administered subcutaneously. The animals were killed at 12:00 h in proestrus, and the ovaries processed for paraffin embedding.

\section{Histological procedures and determination of the labeling index}

The ovaries were serially sectioned (5 $\mathrm{m}$ thick) and placed on poly-L-lysine-coated slides. Unstained slides showing growing follicles, preovulatory follicles, or corpora lutea of the current cycle were selected for inmunohistochemistry. The OSE was divided into three areas: i) OSE covering growing follicles from class 1 onward $(>275 \mu \mathrm{m}$ in diameter), ii) OSE covering corpora lutea of the current cycle, and iii) OSE covering ovarian stroma, including regressing corpora lutea of previous cycles and follicles smaller than class 1. Additionally, the epithelium of the inner aspect of the ovarian bursa, representing extraovarian mesothelium was also scored. In each day of the cycle five different follicles or corpora lutea per rat, and at least five sections per follicle/corpora lutea were immunostained and used for labeling index determination. In pregnant rats, OSE covering corpora lutea of pregnancy (five corpora lutea per rat and five sections per corpus luteum) where analysed. In indomethacin-treated rats, two types of newly formed corpora lutea were found; corpora lutea showing rupture at the apex, and corpora lutea lacking rupture or ruptured at the basolateral sides. Both of these types of corpus lutea showed unruptured OSE (Gaytán et al. 2003) . At least 3 different corpora lutea of each type per rat and five sections per corpus lutea were inmmunostained and used for labeling index determination. The integrity of the OSE in unruptured or abnormally ruptured follicles was confirmed by exhaustive examination of the remaining serial sections stained with hematoxylin and eosin. In rats injected daily with BrdU (cumulative BrdU-labeling), the OSE covering preovulatory follicles (showing cumulative labeling related to follicle growth during the cycle) and the OSE covering carpora lutea of the current cyle (showing cumulative labeling related to ovulation repairing during luteinization) were considered. Five preovulatory follicles and corpora lutea per rat and five sections per follicle or corpus luteum were immunostained. The labeling index was determined by counting the number of OSE cells (labeled and unlabeled) in each OSE area, with the $\times 100$ objective, and expressed as the percentage of labeled OSE cells. Statistical analysis was performed by the Student-ttest or ANOVA followed by the Student-Newman-Keuls method for multiple comparison among means. Significance was considered at the $\boldsymbol{P} \leq 0.05$ level.

\section{Immunohistochemistry of DNA-incorporated BrdU}

DNA-incorporated BrdU was detected by immunohistochemistry with monoclonal anti-BrdU antibodies, following previously described methods (Gaytán et al. 1996).
Briefly, DNA denaturation was carried out by incubating the sections with $0.5 \mathrm{~N} \mathrm{HCl}$ in $0.05 \mathrm{M}$ PBS for $10 \mathrm{~min}$ at $4{ }^{\circ} \mathrm{C}$ and with $2 \mathrm{~N} \mathrm{HCl}$ in $0.05 \mathrm{M}$ PBS for $10 \mathrm{~min}$ at room temperature. After washing and rehydration, the sections were incubated overnight with the anti-BrdU antibody (1:800) and thereafter, processed by the avidin-biotin-peroxidase complex method (Vector, Burlingame, CA, USA) following manufacturers intructions.

\section{Immunohistochemistry of estrogen receptor $\alpha$ (ERa) and progesterone receptor}

Two cycling rats per day of the estrous cycle were killed at 10:00 h. Two additional rats were killed at 21:00 h on the evening of proestrus, after the preovulatory luteinising hormone surge, to study transient expression of PR in preovulatory follicles (Park and Mayo 1991). The ovaries were fixed in $4 \%$ buffered formaldehyde and embedded in paraffin. Immunostainings were performed in dewaxed and hydrated $3 \mu \mathrm{m}$-thick sections. Previous immunocytochemical and in situ hybridization studies have reported that the rat ovarian surface epithelium express ER $\alpha$, but not ERß (Sar and Welsch 1999, Pelletier et al. 2000). For the study of ER $\alpha$ expression, the monoclonal mouse antihuman $E R \alpha$, clone 1D5 (Dakocytomation, Glostrup, Denmark) diluted 1:50, and the LSAB + technique (Dakocytomation, Glostrup, Denmark) were used, following manufacturers instructions. For the study of PR expression, the monoclonal mouse anti-human PR clone PR10A9 (Immunotech, Marseille, France) diluted 1:8000, and the Avidin Biotin Peroxidase complex (ABC) technique (Vector, Burlingame, CA, USA) were used. This PR antibody reacts with both PRA and B subtypes (Szekeres et al. 1994). Following previously established criteria (Sánchez-Criado et al. 2004), several dilutions of the PR10A9 monoclonal antibody were tested in the ovary and in simultaneously processed tissue samples of the rat uterus, these were used as positive controls (data not shown). The optimal dilution was established at 1:8000 because it gave the highest intensity of nuclear staining with the lowest background and cytoplasmic staining, since only nuclear immunostaining was considered as specific. Substitution of the specific primary antibodies by mouse ascitic fluid at the same dilution as the specific primary antibodies, where used as a negative control. Counterstaining was performed with Mayer's hematoxylin.

\section{Results}

\section{BrdU pulse-labeling during the estrous cycle and pregnancy}

The OSE covering ovarian stroma, including small growing follicles and structurally regressing corpora lutea, showed negligible BrdU labeling (Fig. 1a). Labeled cells were only occasionally found (labeling index $<0.1 \%$ ) and counts were not performed. In those areas covering regressing corpora lutea of the previous cycle, still 


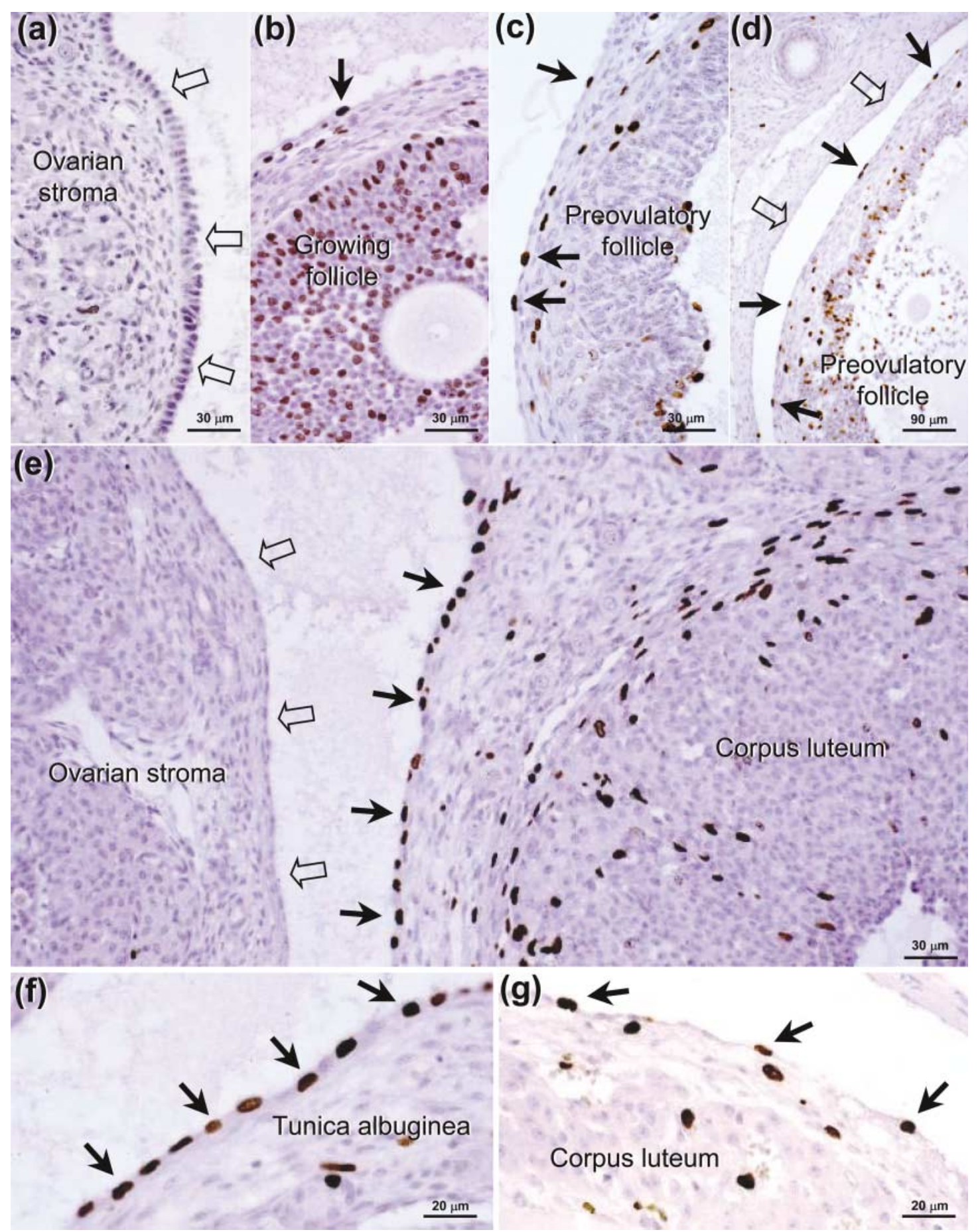

Figure 1 Proliferative activity of the OSE throughout the estrous cycle. OSE areas with (black arrows) and without (empty arrows) BrdU-labeled cells can be observed. (a) Quiescent OSE covering ovarian stroma. (b) OSE covering a class 1 growing follicle at 07:00 h in estrus. (c,d) OSE covering preovulatory follicles at 18:30 h in proestrus and 02:00 h in estrus, respectively. (e) At 07:00 h in estrus, abundant BrdU-labeled cells in the OSE covering a newly-formed corpus luteum, facing an area of quiescent OSE covering ovarian stroma. (f) Higher magnification of OSE covering a newly-formed corpus luteum. (g) OSE covering a corpus luteum in metestrus (Hematoxylin counterstaining).

protruding in the ovarian surface, OSE cells range from flat to cuboidal. In areas covering ovarian stroma or advanced regressing corpora lutea, OSE cells showed variable morphology, ranging from cuboidal to columnar
(Fig. 1a). BrdU-labeled cells were also extremely scarce in the extraovarian mesothelium of the ovarian bursa.

An obvious increase in the number of BrdU-labeled cells in the OSE covering growing follicles, occurred 


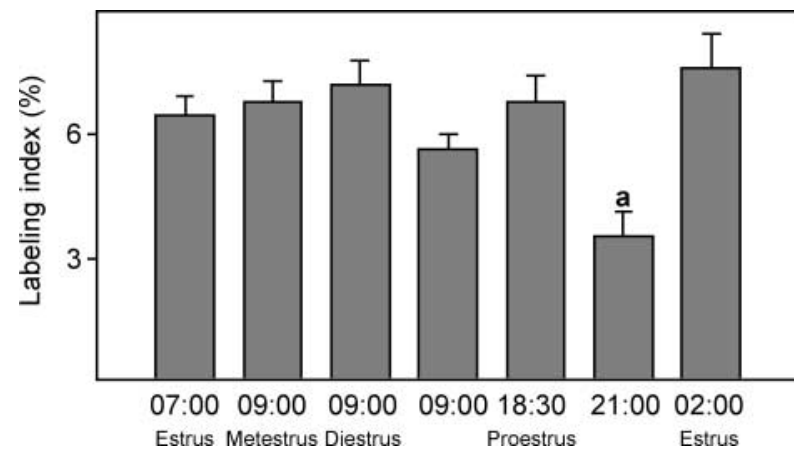

Figure 2 Labeling index of the OSE covering growing and preovulatory follicles during the estrous cycle, after pulse-labeling BrdU treatment. Significant differences $(P<0.05)$ vs the previous value; ANOVA and Student-Newman-Keuls method for $n=5$.

during the transition from proestrus to estrus. On the morning of estrus, the OSE covering class 1 follicles showed a labeling index of about $6 \%$ (Figs $1 \mathrm{~b}$, and 2 ) that remained nearly constant throughout follicle growth during metestrus (covering follicle classes 2 and 3), diestrus (covering class 4 follicles), the morning of proestrus (covering class 5 follicles), and at 18:30 h on the evening of proestrus (covering preovulatory follicles) (Figs 1c and 2). A slight, but significant, decrease in the labeling index was found at 21:00 h on the evening of proestrus (Fig. 2), after the preovulatory luteinising hormone surge, that happens at about 18:30 h in our colony (Gaytán et al. 1997). The labeling index increased again in early estrus (at 02:00 h) inmediately before ovulation (Figs 1d, and Fig. 2). After ovulation, the OSE covering newly formed corpora lutea showed abundant labeled cells during estrus (Figs 1e,f and Fig. 3). Proliferative activity was not limited to the edges of the rupture site, but affected the whole contour of the corpus luteum. The labeling index was about 50\% (Fig. 3) in estrus, showed significant decreases during metestrus (Fig. 1g and Fig. 3) and diestrus (13\% and $3 \%$, respectively) returning to basal levels by proestrus (Fig. 3). The rupture site was covered by epithelium

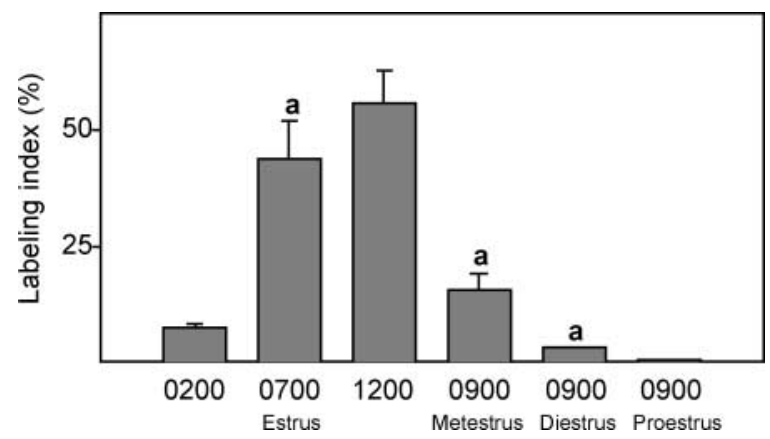

Figure 3 Labeling index of the OSE covering newly-formed corpora lutea during the estrous cycle, after pulse-labeling BrdU treatment. The labeling index of the OSE covering preovulatory follicles immediately before ovulation (at 02:00 $\mathrm{h}$ in estrus) is also indicated as a reference. Significant differences $(P<0.05)$ vs the other groups; ANOVA and Student-Newman-Keuls method for $n=5$. by diestrus. During follicle growth and luteinization, OSE cells progressively changed from cuboidal (covering small follicles) to flat (covering corpus luteum). During pregnancy, the OSE showed extremely flat cells while BrdUlabeled cells were only occasionally found.

\section{Indomethacin-treated rats}

The OSE covering newly formed corpora lutea in indomethacin-treated rats showed abundant BrdU-labeled cells on the morning of estrus (Figs $4 a, b, c$ ), and the labeling index (about 50\%) was equivalent to that of vehicletreated rats, irrespective of the occurrence of rupture or not of the OSE (Figs 4a,b,c and Fig. 5).

\section{Cumulative BrdU-labeling}

On the morning of proestrus, the OSE covering preovulatory follicles showed abundant BrdU labeled cells (Fig. $4 d$ ). The labeling index, as an estimate of the cumulative proliferative activity related to follicle growth during the estrous cycle, was about 33\% (Fig. 6). Most epithelial cells in the OSE covering corpus luteum of the current cycle were BrdU-labeled (Figs 4e,f). The labeling index, as an estimate of the cumulative proliferative activity related to ovulation rupture repair/luteinization, was about $75 \%$ (Fig. 6). Otherwise, the number of BrdU labeled cells in the OSE covering the ovarian stroma, as well as in the extraovarian epithelium of the ovarian bursa, was negligible (Figs 4d,e).

\section{Immunohistochemistry of ER $\alpha$ and PR}

In the ovary of cycling rats, $\mathrm{ER} \alpha$ immunoreactivity was observed in the nuclei of the theca of growing follicles, interstitial gland and OSE (Fig. 7a) throughout the estrous cycle, whereas granulosa cells and corpora lutea were negative. Immunostaining of the OSE was not regionalized, and the nuclei of OSE cells were evenly immunostained in both proliferating and quiescent areas. On the contrary, PR immunoreactivity was absent in the rat ovary, except at 21:00 h on the evening of proestrus, when intense immunostaining was observed in the granulosa cells of preovulatory follicles (Fig. 7b). This, together with initial preovulatory changes such as cumulus dispersion and edematization of the apex, gave evidence for the occurrence of a previous preovulatory LH surge. However, OSE cells covering preovulatory follicles remained negative for PR (Fig. 7b).

\section{Discussion}

The OSE shows local variations in its morphology and proliferative activity, related to the cyclic changes in the underlying ovarian structure. The data of this study allow the proposal and characterization of a cycle of the OSE outlined in Fig. 8. Consequently, three different areas of the OSE, showing relevant differences in its morphology, 


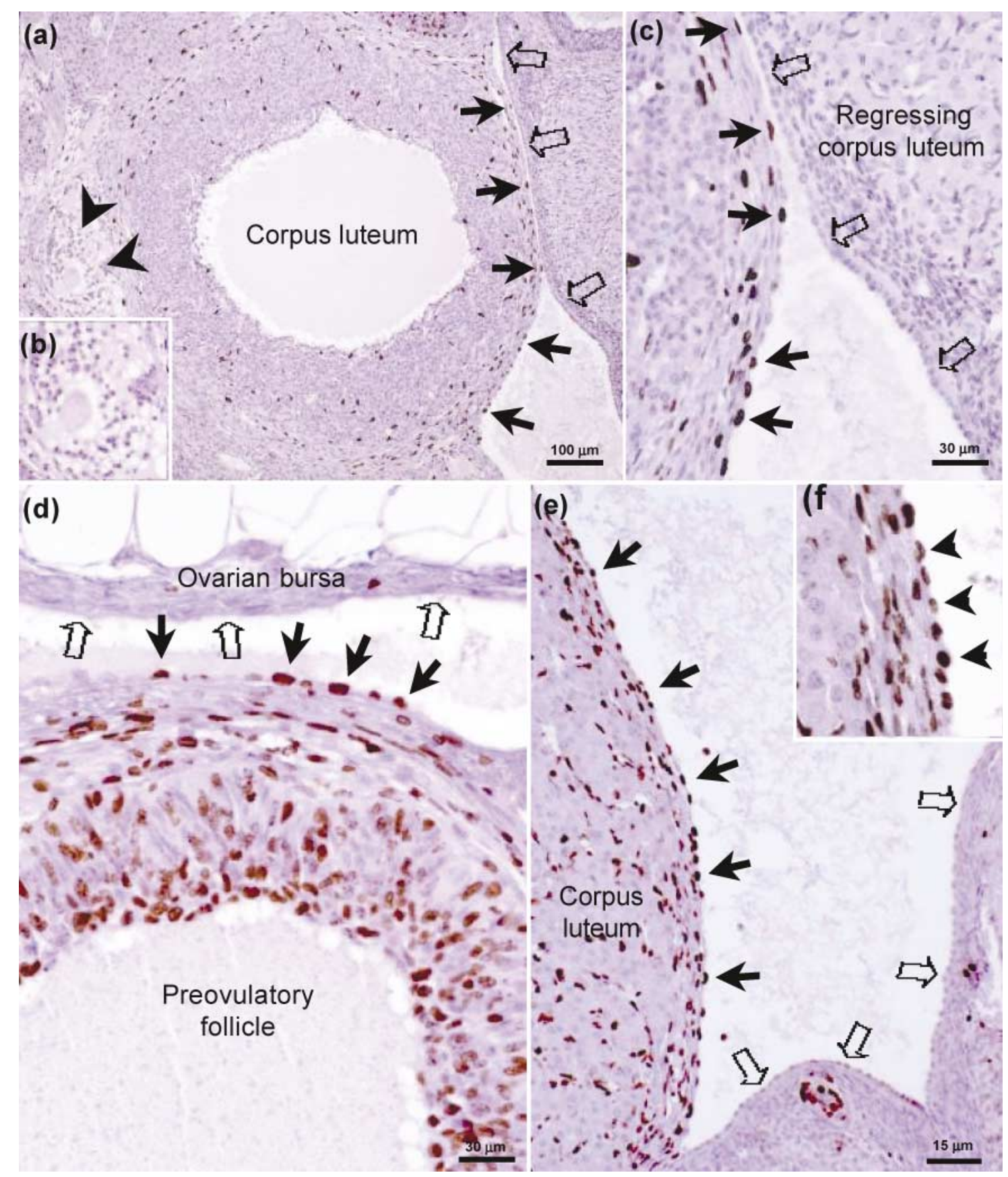

Figure $4(a, b, c)$ Proliferative activity on the morning of estrus in rats treated with indomethacin. A newly-formed corpus luteum, showing release of the oocyte at the basal side (arrowheads in (a), and at higher magnification in (b)), showing abundant BrdU-labeled cells in the unruptured OSE at the apex (black arrows), facing an area of OSE covering a regressing corpus luteum (empty arrows), lacking BrdU-labeled cells. (c) Higher magnification of the same area in an adjacent section. (d,e,f) Cumulative proliferative activity in proestrus. BrdU-labeled cells (black arrows) are abundant in OSE areas covering preovulatory follicles (d) or corpora lutea of the current cycle (e,f), and absent (empty arrows) in the epithelium of the ovarian bursa (d), or in the OSE covering ovarian stroma (e) (Hematoxylin counterstaining).

proliferative activity and probably, in other functional characteristics, are present at any time of the estrous cycle: i) the OSE covering growing follicles, ii) the OSE covering $\mathrm{CL}$ of the current cycle, both in the proliferative phase, and iii) the remaining OSE in the quiescent phase. The dependence of the morphological features and prolif- erative activity of the OSE on the underlying ovarian structure, strongly suggests that its functional status is dependent on local microenviromental rather than on systemic factors. This could explain, at least in part, the controversial data about the effects of reproductive hormones on OSE proliferative activity in in vivo vs in vitro studies. 


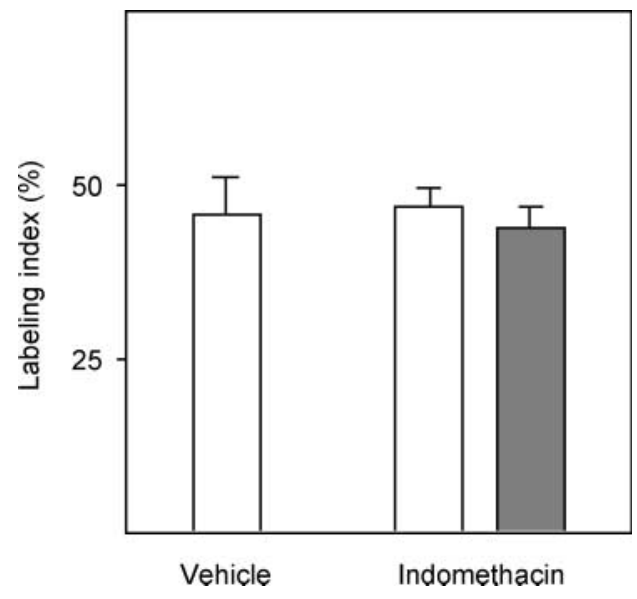

Figure 5 Labeling index of the OSE covering newly-formed corpora lutea in estrus, after pulse-labeling BrdU treatment in rats treated with vehicle or indomethacin. Empty bars correspond to corpora lutea with rupture at the apex, whereas the filled bar corresponds to corpora lutea without rupture of the OSE.

Whereas in vivo studies have reported that gonadotropic hormones affect OSE cell proliferation (Bai et al. 2000, Murdoch and van Kirk 2002), some in vitro studies with isolated OSE cells have found a lack of mitogenic effects of gonadotropic hormones (Wright et al. 2002). The data of this study suggests that local factors, most likely released by growing follicles and corpora lutea, are those stimulating OSE cell proliferation. In this context, it can be expected that isolated OSE cells are unresponsive to reproductive hormones, just as the in vivo OSE in areas covering regressing corpora lutea or ovarian stroma. Several growth factors such as hepatocyte growth factor (HGF) (Hess et al. 1999), and stem cell factor (SCF) (Parrot et al. 2000), have been reported to regulate OSE cell proliferation in vitro. The expression of receptors for these

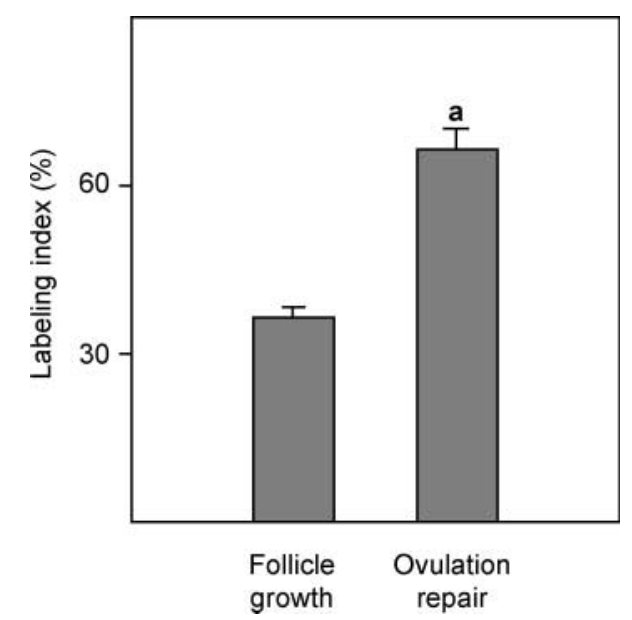

Figure 6 Cumulative labeling index of the OSE covering preovulatory follicles (related to follicle growth) or corpora lutea of the current cycle (related to ovulation repair) in proestrus. $(P<0.01$, Student $t$-test for $n=5)$. growth factors in the OSE (Hess et al. 1999, Parrot et al. 2000), suggests that autocrine and paracrine routes are likely to be involved.

Steroid hormones released by the follicle and/or the corpus luteum are strong candidates for factors which regulate the proliferative activity of OSE cells. In general, like in many other tissues, several studies have reported that estradiol stimulates OSE cell proliferation (Murdoch and van Kirk 2002, Ho 2003), whereas progesterone has been reported to inhibit estrogen-mediated OSE cell proliferation (Murdoch \& van Kirk 2002). The proposed inhibitory effects of progesterone are in line with the low proliferative activity of OSE cells found in this study during pregnancy. However, due to the existence of OSE areas showing different proliferative activity at the same phase of the estrous cycle, a clear correlation between serum hormone concentrations and OSE cell proliferation cannot be established from the present data. Additional studies involving supplementation/supression of steroid hormones are needed to achieve definitive conclusions. In spite of the abundant data in the literature, it is unclear whether estradiol and progesterone effects on the OSE are direct or mediated by local factors. Interestingly, the proliferative response of OSE cells to estrogen is enhanced when epithelial cells are grown together with ovarian stromal cells (Bai et al. 2000), suggesting the existence of stromal-epithelial interactions. Moreover, it has been reported that estrogens do not have mitogenic effects on isolated OSE cells (Karlan et al. 1995, Wright et al. 2002). In this study, immunostaining of OSE cells for $\mathrm{ER} \alpha$ was not regionalized, and the expression of $\mathrm{ER} \alpha$ was equivalent (at least qualitatively) in both proliferating and quiescent areas. This suggests that the proposed effects of estrogens on OSE cell proliferation are mediated by local microenvironmental factors. Similarly, the absence of PR expression in the rat OSE strongly suggests that the proposed progesterone effects on OSE cell proliferation are indirect. However, the possibility of non-genomic responses to steroid hormones, independent of classic genomic steroid receptors (Bramley 2003) cannot be discarded.

Some previous studies have analysed the proliferative activity of the OSE in immature gonadotropin-primed rats and mice (Beller et al. 1995, Hess et al. 1999). It is worthy to note that in immature animals, the development of a large cohort of follicles (about 50) and subsequent corpora lutea is stimulated by equine chorionic gonadotrophin (eCG) and human chorionic gonadotrophin (hCG) treatment. In addition, the ovary considerably enlarges as a consequence of superovulation. Accordingly, large areas of the OSE should enter into the proliferative phase in immature gonadotropin-primed rats. This would likely lead to overestimation of the proliferative response of the OSE to gonadotropin treatment when compared with adult cycling rats, and could mask the existence of quiescent, non-proliferating areas. In adult animals, the increasing volume of growing follicles and newly formed corpora lutea is compensated by the loss of volume of several 

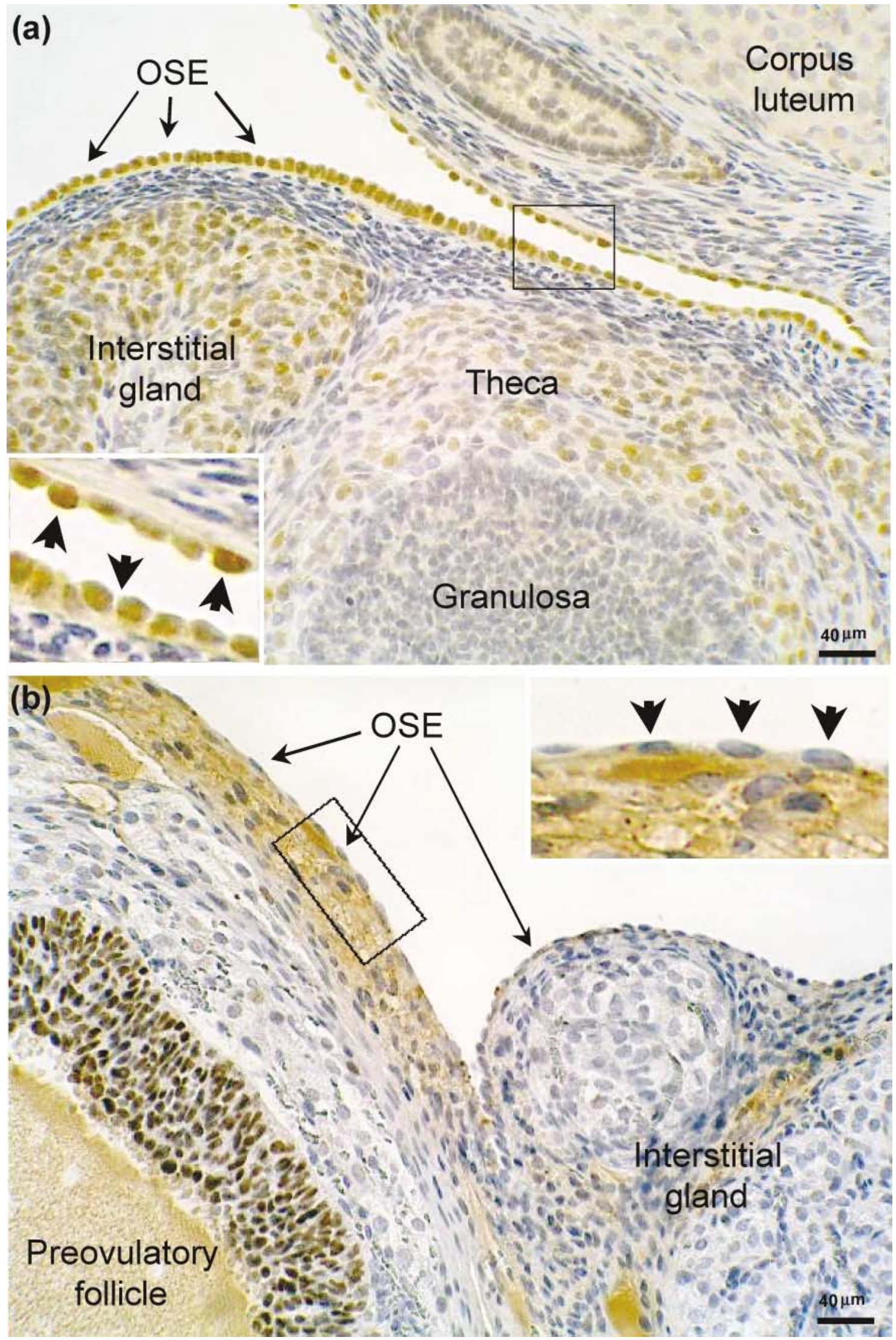


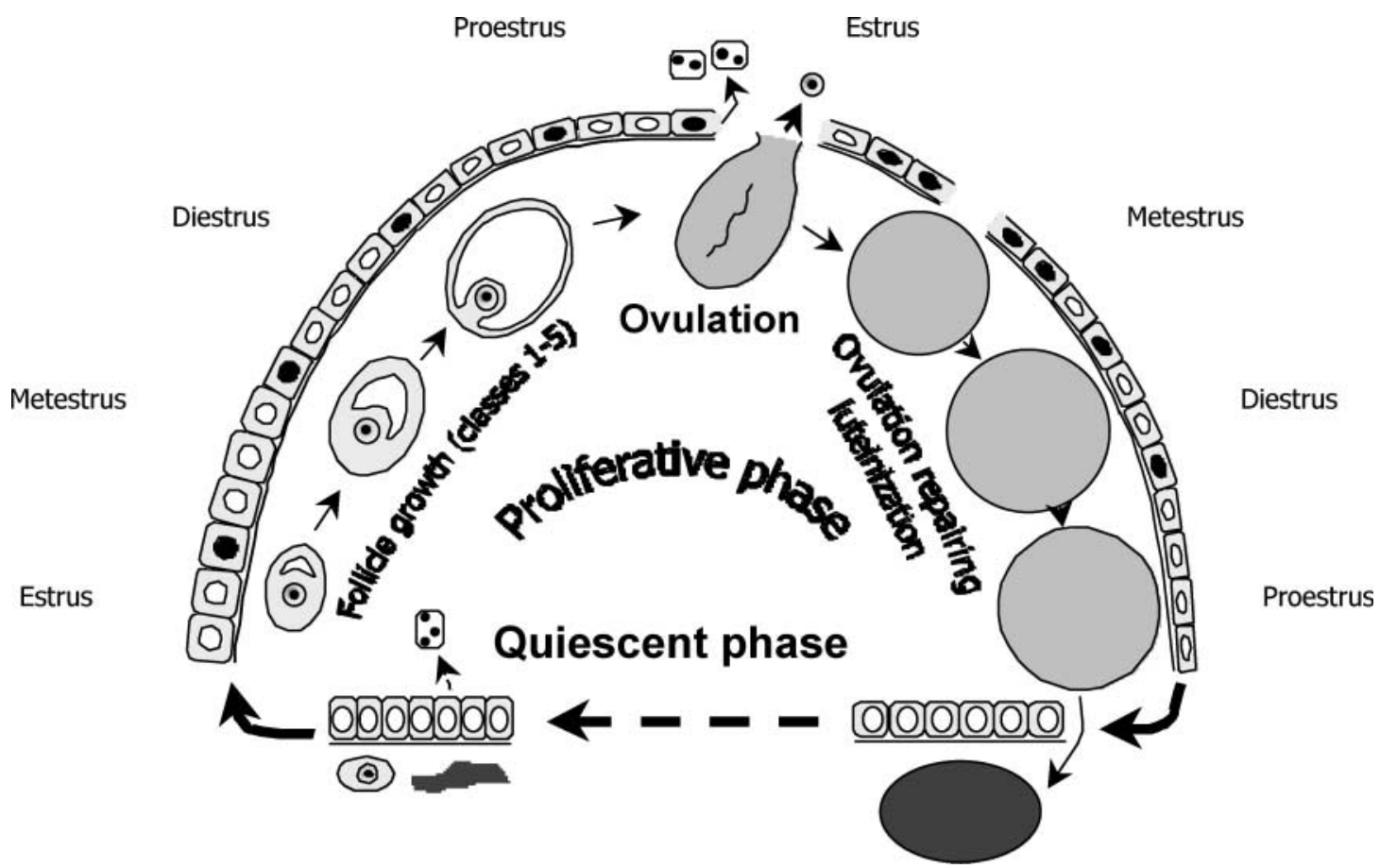

Figure 8 Schematic drawing of the cycle of the OSE. The cycle consists of two phases: a proliferative phase and a quiescent phase. The proliferative phase lasts for two estrous cycles in parallel to the development of the follicle-corpus luteum unit. During the first estrous cycle, OSE cells covering growing follicles enter into the proliferative phase in coincidence with the appearance of class 1 follicles during the transition from proestrus to estrus. These follicles are those destined to ovulate at the end of the current estrous cycle. A nearly constant labeling index of OSE cells of about $7 \%$ is maintained from estrus to the evening of proestrus, in parallel to follicle growth from class 1 to class 5 (preovulatory) follicles (epithelial cells with filled nuclei correspond to proliferating cells). About 1/3 of the total proliferative activity occurs during the first estrous cycle, in relation to follicle growth. During the second estrous cycle, rupture of the OSE at the site of ovulation occurs on early estrus. This is followed by an intense proliferative activity in the OSE covering the newly-formed corpus luteum during estrus (labeling index of about $50 \%$ ), that decreased thereafter during metestrus (labeling index of about $15 \%$ ) and diestrus (labeling index of about $3 \%$ ), and falling to basal levels by proestrus. About $2 / 3$ of the total proliferative activity occurs during the second estrous cycle, in relation to ovulation repair/luteinization. During the proliferative phase, the shape of OSE cells progressively changes from cuboidal to flat. The transition from proestrus to estrus marks the beginning of the quiescent phase, which lasts for a variable number of estrous cycles depending on the eventual recruitment of new growing class 1 follicles in that area. The proliferative activity is negligible, and the shape of OSE cells progressively changes from flat to cuboidal or columnar. If pregnancy occurs, the quiescent phase is enlengthened, as no significant proliferative activity is associated to the development of the $\mathrm{CL}$ of pregnancy. Epithelial cells are eliminated by apoptosis (cells with fragmented nuclei) at the site of rupture and possibly, at a low rate during the quiescent phase.

generations of regressing corpora lutea of previous cycles, this occurs at each transition from proestrus to estrus, triggered by the preovulatory PRL surge. In this way, the ovarian volume, and hence the ovarian surface, does not undergo significant changes throughout the estrous cycle.

The data of this study question the fate of OSE cells throughout the estrous cycle. As the ovarian surface did not show relevant changes throughout the cycle, a mechanism responsible for the elimination of OSE cells should exist to compensate for the abundant OSE cell proliferation. The only obvious mechanism for OSE cell deletion, the demise of cells at the rupture site during ovulation (affecting a small area with respect to the preovulatory follicle protrusion) is likely outnumbered by the proliferative activity of OSE cells before and after OSE rupture, involving the whole follicle/corpus luteum contour facing the ovarian surface. In this study, the presence of apoptotic cells was estimated by morphological criteria. We have previously reported (Gaytán et al. 1998) that no significant differences exist for counts of apoptotic cells between morphological evaluation and immunostained cells with the TdT-mediated dUTP digoxigenin nick end labelling

Figure 7 Immunohistochemical detection of ER $\alpha$ (a) and PR (b) in the ovary of cycling rats, at 10:00 h in diestrus (a) and at 21:00 $\mathrm{h}$ in proestrus (b). ER $\alpha$ immunoreactivity can be observed in the nuclei of the theca, interstitial gland and OSE, whereas the granulosa and the corpus luteum are negative. Nuclear immunostaining of OSE cells is shown at higher magnification in the inset, corresponding to the framed area in (a). Intense PR immunoreactivity can be observed exclusively in the nuclei of granulosa cells in preovulatory follicles. The edematized area at the apex (asterisks) shows non-specific staining. Negative OSE cell nuclei are shown at higher magnification in the inset, corresponding to the framed area in (b) (Hematoxylin counterstaining). 
(TUNEL) method in the ovary. In the present study, apoptotic figs were extremely scarce on histological examination. Possibly, apoptotic cells are exfoliated to the bursal cavity and phagocytosed by resident peritoneal macrophages. Although definitive conclusions cannot be achieved from the present data, we propose herein that cell loss by low rate apoptosis during the quiescent phase should be the mechanism compensating OSE cell proliferation. Progesterone and estradiol have been reported to modulate OSE cell apoptosis (Murdoch \& van Kirk 2002, Ho 2003), although the in vivo regulation of OSE cell apoptosis is not fully understood.

As rupture of the OSE at the apex is an obligate component of ovulation, most attention on the OSE proliferative activity has been devoted to ovulatory wound repair. However, the data of this study indicate that a substantial part of the total OSE proliferation was previous to ovulation rupture, and related to follicle growth. Although not mutually exclusive, the 'incesant ovulation' (Fathalla 1971) and 'gonadotropin stimulation' (Cramer and Welch 1983) hypotheses (considering ovulatory wound repair and gonadotropin stimulation, respectively, as the main factors favouring mutagenic events) have been independently considered. The data of this study are consistent with both hypotheses. Data from cumulative BrdU-labeling indicate that about $1 / 3$ of the total OSE cell proliferation was related to follicle growth (therefore indirectly related to gonadotropin-dependent follicle growth), and about $2 / 3$ to ovulation repair/luteinization.

Prostaglandins are essential factors during the ovulatory process (Tsafriri et al. 1993) and some of the changes that happen in the OSE during ovulation have been reported to be mediated by prostaglandins (Ackerman and Murdoch 1993, Gaytán et al. 2002). In this context, we analysed the proliferative response of the OSE after ovulation in rats treated with indomethacin. The equivalent proliferative activity of OSE cells in vehicle or indomethacin-treated rats indicates that post-ovulatory proliferative activity of OSE cells was neither mediated by prostaglandins, nor affeted by indomethacin treatment through prostaglandin-independent routes. Data from indomethacin-treated rats also provided information on the mechanism of OSE cell proliferation at the site of ovulation. Although rupture of the integrity of the OSE has been considered as the main factor determining OSE cell proliferation after ovulation, the data of this study indicate that ovulation-triggered OSE cell proliferation was not directly due to the occurrence of rupture of the OSE but to ovulation-related events, as indicated by the similar proliferative activity in newly-formed corpora lutea, in which rupture at the apex did not occur. It is also supported by the extent of the proliferative activity that was not limited to the edges of the rupture sites, but affected the whole corpus luteum contour.

In summary, the existence of local changes in the morphology and proliferative activity of the OSE, related to cyclic changes in the underlying ovarian structure, demonstrates the existence of a cycle of the OSE, and suggest that the functional status of OSE cells is regulated by local microenviromental, rather than by systemic factors.

\section{References}

Ackerman RC \& Murdoch WJ 1993 Prostaglandin-induced apoptosis of ovarian surface epithelial cells. Prostaglandins 45 475-485.

Adams AT \& Auersperg N 1983 Autoradiographic investigation of estrogen binding in cultured rat ovarian surface epithelial cells. Journal of Histochemistry and Cytochemistry 31 1321-1325.

Auersperg N, Wong AST, Choi KC, Kang SK \& Leung PCK 2001 Ovarian surface epithelium: biology, endocrinology, and pathology. Endocrine Reviews 22 255-288.

Bai W, Oliveros-Saunders B, Wang Q, Acevedo-Duncan ME \& Nicosia SV 2000 Estrogen stimulation of ovarian surface epithelial cell proliferation. In Vitro Cell \& Developmental Biology - Animal 36 657-666.

Beller U, Haimovitch R \& Ben-Sasson S 1995 Periovulatory multifocal mesothelial proliferation: a possible association with malignant transformation. International Journal of Gynecological Cancer 5 306-309.

Bjersing L \& Cajander S 1975 Ovulation and the role of the ovarian surface epithelium. Experientia 31 605-608.

Bramley T 2003 Non-genomic progesterone receptors in the mammalian ovary: some unresolved issues. Reproduction 125 3-15.

Cramer DW \& Welch WR 1983 Determinants of ovarian cancer risk II. Inferences regarding pathogenesis. Journal of the National Cancer Institute 71 717-721.

Davies BR, Finningan DS, Smith SK \& Ponder BA 1999 Administration of gonadotropins stimulates proliferation of normal mouse ovarian surface epithelium. Gynecological Endocrinology 13 $75-81$.

Fathalla MF 1971 Incessant ovulation- a factor in ovarian neoplasia? Lancet 2163.

Gaytán F, Morales C, Bellido C, Aguilar E \& Sánchez-Criado JE 1996 Proliferative activity in the different ovarian compartmentsin cycling rats estimated by the 5bromodeoxyuridine technique. Biology of Reproduction 54 1356-1365.

Gaytán F, Bellido C, Morales C, Aguilar E \& Sánchez-Criado JE 1997 Follicular growth pattern in cycling rats from late pro-oestrus to early oestrus. Journal of Reproduction and Fertility 110 153-159.

Gaytán F, Bellido C, Morales C \& Sánchez-Criado JE 1998 Both prolactin and progesterone in proestrus are necessary for the induction of apoptosis in the regressing corpus luteum of the rat. Biology of Reproduction 59 1200-1206.

Gaytán F, Tarradas E, Morales C, Bellido C \& Sánchez-Criado JE 2002 Morphological evidence for uncontrolled proteolytic activity during the ovulatory process in indomethacin-treated rats. Reproduction 123 639-649.

Gaytán F, Bellido C, Gaytán M, Morales C \& Sánchez-Criado JE 2003 Differential effects of RU486 and indomethacin on follicle rupture during the ovulatory process in the rat. Biology of Reproduction 69 99-105.

Godwin AK, Testa JR \& Hamilton TC 1993 The biology of the ovarian cancer development. Cancer 71 530-536.

Hess S, Gulati R \& Peluso JJ 1999 Hepatocyte growth factor induces rat ovarian surface epithelial cell mitosis or apoptosis depending on the presence or absence of an extracellular matrix. Endocrinology 140 2908-2916.

Hild-Petito S, Stouffer RL \& Brenner RM 1988 Immunocytochemical localization of estradiol and progesterone receptors in the monkey ovary thoughout the menstrual cycle. Endocrinology 123 2896-2905.

Ho SM 2003 Estrogen, progesterone and epithelial ovarian cancer. Reproductive Biology and Endocrinology 1 73-80. 
Karlan BY, Jones J, Greenwald M \& Lagasse LD 1995 Steroid hormone effects on the proliferation of human ovarian surface epithelium in vitro. American Journal of Obstetric and Gynecology 173 97-104.

Kuroda H, Mandai M, Konishi I, Tsuruta Y, Kusakari T, Kariya M \& Fujii S 2001 Human ovarian surface epithelial (OSE) cells espress LH/hCG receptors, and hCG inhibits apoptosis of OSE cells via upregulation of insulin-like growth factor-1. International Journal of Cancer 91 309-315.

Murdoch WJ \& McDonnel AC 2002 Roles of ovarian surface epithelium in ovulation and carcinogenesis. Reproduction $\mathbf{1 2 3}$ $743-750$.

Murdoch WJ, Townsen RS \& McDonnel AC 2001 Ovulation-induced DNA damage in ovarian surface epithelial cells of ewes: prospective regulatory mechanisms of repair/survival and apoptosis. Biology of Reproduction 65 1417-1424.

Murdoch WJ \& van Kirk EA 2002 Steroid hormonal regulation of proliferative, p53 tumor suppressor, and apoptotic responses of sheep ovarian surface epithelial cells. Molecular and Cellular Endocrinology 186 61-67.

Okada A, Ohta Y, Buchanan DL, Sato T, luone S, Hiroi $\mathbf{H}$, Muramatsu M \& Iguchi T 2002 Changes in ontogenic expression of estrogen receptor alpha and not of estrogen receptor beta in the female rat reproductive tract. Journal of Molecular Endocrinology 28 87-97.

Osterholzer HO, Streibel EJ \& Nicosia SV 1985 Growth effects of protein hormones on cultured rabbit ovarian surface epithelial cells. Biology of Reproduction 33 247-258.

Park OK \& Mayo KE 1991 Transient expression of progesterone receptor messenger RNA in ovarian granulosa cells after the preovulatory luteinizing hormone surge. Molecular Endocrinology 5 967-978.

Parrot JA, Kim G \& Skinner MK 2000 Expression and action of kit ligand/stem cell factor in normal human and bovine ovarian surface epithelium and ovarian cancer. Biology of Reproduction 62 1600-1609.

Pelletier G, Labrie C \& Labrie F 2000 Localization of oestrogen receptor $\alpha$, oestrogen receptor $\beta$, and androgen receptors in the rat reproductive organs. Journal of Endocrinology $\mathbf{1 6 5}$ $359-370$.
Rawson JMR \& Espey LL 1977 Concentration of electron dense granules in the rabbit ovarian surface epithelium during ovulation. Biology of Reproduction 17 561-566.

Sánchez-Criado JE, Martín de las Mulas J, Bellido C, Tena-Sempere M, Aguilar R \& Blanco A 2004 Biological role of pituitary estrogen receptors $E R \alpha$ and $E R \beta$ on progesterone receptor expression and action and on gonadotropin and prolactin secretion in the rat. Neuroendocrinology 79 247-258.

Sar M \& Welsch F 1999 Differential expression of estrogen receptor$\beta$ and estrogen receptor- $\alpha$ in the rat ovary. Endocrinology 140 967-971.

Stewart SL, Querec TD, Gruver BN, O'Hare B, Babb JS \& Patriotis C 2004 Gonadotropin and steroid hormones stimulate proliferation of the rat ovarian surface epithelium. Journal of Cell Physiology $198119-124$.

Syed V, Ulinski G, Mok SC, Yiu GK \& Ho SM 2001 Expression of gonadotropin receptor and growth responses to key reproductive hormones in normal and malignant human ovarian surface epithelial cells. Cancer Research 61 6768-6776.

Szekeres G, Lutz Y, Le Tourneau A \& Delaage M 1994 Steroid hormone receptor immunostaining on paraffin sections with microwave heating and trypsin digestion. Journal of Histochemistry $\mathbf{1 7}$ $321-324$.

Tsafriri A, Chun SY \& Reich R 1993 Follicular rupture and ovulation. In The Ovary, pp 228-243. Eds EY Adashi \& PCK Leung. New York: Raven Press.

Wright JW, Toth-Fejel S, Stouffer RL \& Rodland KD 2002 Proliferation of rhesus ovarian surface epithelial cells in culture: lack of mitogenic response to steroid or gonadotropic hormones. Endocrinology 143 2198-2207.

Zheng W, Magid MS, Kramer EE \& Chen YT 1996 Follicle-stimulating hormone receptor expressed in human ovarian surface epithelium and fallopian tube. American Journal of Pathology 148 47-53.

Received 2 July 2004

First decision 16 August 2004

Revised manuscript received 29 October 2004

Accepted 5 January 2005 